Artículos

\title{
Calidad de vida del paciente con enfermedad pulmonar obstructiva crónica*
}

\author{
Quality of Life of Patients with Chronic Obstructive Pulmonary Disease \\ Qualidade de vida de pacientes com doença pulmonar obstrutiva crônica
}

\author{
Luisa Fernanda Achury Beltrán ${ }^{\text {a }}$ \\ Pontifica Universidad Javeriana, Colombia \\ achuryl@javeriana.edu.co \\ ORCID: https://orcid.org/0000-0002-7637-3722 \\ Pilar García Peñuela \\ Pontifica Universidad Javeriana, Colombia \\ ORCID: https://orcid.org/0000-0002-7637-3722
}

DOI: https://doi.org/10.11144/Javeriana.ie23.cvpe

Recibido: 21 Agosto 2020

\section{Resumen:}

Los pacientes que padecen de patologías crónicas, como la enfermedad pulmonar obstructiva, experimentan diversos cambios en su estado de salud, que los lleva a modificar su percepción de bienestar. Por ello, la evaluación de la calidad de vida se hace necesaria. Objetivo: describir, de forma global y por dimensiones, la calidad de vida de sujetos que padecen de Enfermedad Pulmonar Obstructiva Crónica (EPOC), que acudieron a la consulta externa de una institución hospitalaria en el primer semestre de 2018 en Bogotá. Método: las características sociodemográficas y la calidad de vida relacionada con salud se midieron en un estudio descriptivo transversal. La muestra estuvo conformada por 80 pacientes. El análisis se fundamentó en la aplicación de frecuencias y porcentajes para las variables sociodemográficas, y de medidas de tendencia central para la variable principal de la investigación. Resultados: los pacientes con EPOC reportaron una edad media de 73 años, fueron predominantemente mujeres, casadas, con bajo nivel de escolaridad y con una media en la calidad de vida de 36,67. La subescala más afectada fue la de actividad y la menos comprometida fue la dimensión de impacto. Conclusiones: la calidad de vida relacionada con la salud es un indicador de las consecuencias que tiene la EPOC sobre la ejecución de las diferentes actividades físicas y sociales de los pacientes, por ello, su evaluación se convierte en un aspecto fundamental en la valoración e intervención de dicha población.

Palabras clave: calidad de vida, pacientes, enfermedades respiratorias, enfermería.

\section{Abstract:}

Patients suffering from chronic pathologies, such as obstructive pulmonary disease, experience various changes in their health state, which leads them to modify their perception of well-being. Therefore, an evaluation of their quality of life is necessary. Objective: to describe, globally and by dimensions, the quality of life of people suffering from Chronic Obstructive Pulmonary Disease (COPD) who attended the outpatient consultation of a hospital institution in the first semester of 2018 in Bogotá. Method: sociodemographic characteristics and health-related quality of life were measured in a descriptive cross-sectional study. The sample consisted of 80 patients. The analysis was based on applying frequencies and percentages for sociodemographic variables and measures of central tendency for the primary variable of the research. Results: COPD patients reported a mean age of 73 years. They were predominantly women, married, with a low education level and a mean quality of life of 36.67 . The most affected subscale was activity, and the least compromised was the impact dimension. Conclusions: health-related quality of life is an indicator of COPD's consequences on the performance of different physical and social activities of patients; therefore, its evaluation becomes a fundamental aspect in the assessment and intervention of said population.

Keywords: quality of life, patients, respiratory diseases, nursing.

\section{Resumo:}

Os pacientes portadores de patologias crônicas, como a doença pulmonar obstrutiva, vivenciam diversas alterações no estado de saúde, o que os leva a modificar sua percepção de bem-estar. Portanto, a avaliação da qualidade de vida é necessária. Objetivo: descrever, globalmente e por dimensões, a qualidade de vida de sujeitos com Doença Pulmonar Obstrutiva Crônica (DPOC), que compareceram à consulta ambulatorial de uma instituição hospitalar no primeiro semestre de $2018 \mathrm{em}$ Bogotá. Método: as características sociodemográficas e a qualidade de vida relacionada à saúde foram mensuradas em um estudo descritivo transversal. A amostra foi composta por 80 pacientes. A análise alicerçou-se na aplicação de frequências e percentuais para as variáveis

Notas de autor

\footnotetext{
${ }^{a}$ Autora de correspondencia. Correo electrónico: achuryl@javeriana.edu.co
} 
sociodemográficas e de medidas de tendência central para a variável principal da pesquisa. Resultados: Pacientes com DPOC relataram idade média de 73 anos, eram predominantemente mulheres, casadas, com baixa escolaridade e qualidade de vida média de 36,67. A subescala mais afetada foi a de atividade e a menos comprometida foi a dimensão do impacto. Conclusões: a qualidade de vida relacionada à saúde é um indicador das consequências que a DPOC tem no desempenho das diferentes atividades físicas e sociais dos pacientes, portanto, sua avaliação torna-se um aspecto fundamental na valoração e intervenção dessa população.

Palavras-chave: qualidade de vida, pacientes, doenças respiratórias, enfermagem.

\section{Introducción}

Las enfermedades respiratorias generan un fuerte impacto sobre la salud de las personas a nivel global y, desde allí, la EPOC cobra relevancia por las repercusiones físicas y emocionales que se producen en quienes la padecen. De acuerdo con la Global Initiative for Chronic Obstructive Lung Disease (GOLD), esta se refiere a "una enfermedad frecuente, prevenible y tratable que se caracteriza por unos síntomas respiratorios y una limitación al flujo aéreo persistente, los cuales se deben a anomalías de las vías respiratorias y/o alveolares causadas generalmente por una exposición importante a partículas o gases nocivos" (1). La espirometría sigue siendo el paraclínico más relevante para el diagnóstico, cuyo parámetro de corte se refiere a un índice VEF1/ CVF menor a $70 \%$ o al valor inferior del rango establecido para la población a la que pertenece el paciente, en el test con broncodilatador (2).

De acuerdo con Martínez-Luna et al. (3), la EPOC se ubica en la cuarta posición dentro de las causas de mortalidad a nivel mundial y se proyecta que subirá al tercer lugar en las causas de fallecimientos en el 2030. Según los datos publicados por el Instituto de métricas en salud en el 2017, esta enfermedad representó el $5,72 \%$ de las causas globales de mortalidad, mientras que en Colombia generó el 5,9\% del total de muertes reportadas (4), datos similares a los de Gil-Rojas et al. (5), quienes estimaron la prevalencia de la EPOC en Colombia en 5,13\%, dichos reportes denotan la envergadura de la enfermedad y con ello el desafío que implica en términos de la salud a nivel mundial.

La evidencia sobre los efectos de la enfermedad es clara, los pacientes presentan síntomas que actúan como indicadores clínicos asociados al diagnóstico de la patología, como lo son la sensación de falta de aire, la tos crónica, y la presencia de secreciones e infecciones respiratorias frecuentes (1), los cuales se acompañan comúnmente de intolerancia a la actividad, aspectos que comprometen el funcionamiento del paciente y su familia en diferentes dimensiones.

En este sentido, los pacientes reportan su experiencia de enfermedad como una alteración en la vida cotidiana que les impide realizar una planificación de sus actividades, pues estas llegan a depender del estado de salud percibido a diario por parte del paciente (6).

Por esta razón, la calidad de vida cobra relevancia en la valoración que realiza el profesional de enfermería y en el manejo de las personas que padecen la enfermedad, pues los síntomas se asocian a la reducción de la actividad física, la aparición de trastornos emocionales (7) y del sueño (8), compromiso nutricional (9), sarcopenia (10) y disfunción de los músculos periféricos (11), situaciones que afectan el desempeño habitual, laboral y psicosocial de los pacientes.

Debido al impacto de la enfermedad, el concepto de la calidad de vida relacionada con la salud se ha convertido en un fenómeno de estudio relevante y se ha hecho visible en la evaluación de la afección que producen las enfermedades crónicas en las diferentes dimensiones del ser humano. No existe una definición unificada sobre este concepto, sin embargo, para Urzúa es "el nivel de bienestar derivado de la evaluación que la persona realiza de diversos dominios de su vida, considerando el impacto que en estos tiene su salud" (12), de ahí que la medición de este fenómeno resulta relevante en la valoración que realiza el profesional de enfermería al paciente con EPOC, pues permite describir las consecuencias y los cambios en el funcionamiento derivados de su condición de salud y, a partir de allí, generar una planificación individualizada de las actividades de 
cuidado que el paciente requiere para mejorar el control de la enfermedad, y con ello la percepción de bienestar.

En Colombia, algunos autores han aplicado el cuestionario respiratorio de Saint George (SGRQ) para medir la calidad de vida al paciente con EPOC, donde se encontró una puntuación promedio que oscila entre 38 (13) y 48 \% (14); así como para determinar la efectividad de programas de atención integral (15), lo cual soporta el uso de este instrumento.

\section{Método}

Se realizó un estudio descriptivo transversal que estima la calidad de vida de individuos con diagnóstico de EPOC, durante el primer semestre del 2018.

La población estuvo constituida por personas con diagnóstico de EPOC en su historial médico, que asistieron a control por consulta externa en un centro hospitalario en la ciudad de Bogotá para el primer semestre del 2018. El tamaño muestral obtenido fue de 80 pacientes, donde se usó para el cálculo un alfa de 0,05 y una potencia estadística de $80 \%$ con una fórmula para muestras finitas. Se utilizó un muestro no probabilístico por conveniencia.

Como criterios de inclusión se tuvieron en cuenta personas que asistieron a control de consulta externa de un centro hospitalario de alta de complejidad en Bogotá, con diagnóstico de EPOC en la historia clínica y que desearan voluntariamente participar en la investigación. Se excluyeron aquellos pacientes con exacerbaciones durante el último mes o aquellos que en la historia clínica evidenciaran asociación de otras enfermedades pulmonares crónicas, déficit cognitivo o alteraciones de tipo mental que les impidieran el diligenciamiento del instrumento utilizado, que fue el SGRQ para la evaluación de la calidad de vida, el cual está constituido por 50 preguntas que se agrupan en las dimensiones de síntomas, actividad e impacto, con lo que arroja un resultado global y una puntuación de acuerdo con las subescalas.

El instrumento fue creado por Jones, y la traducción y validación al español fue realizada por Ferrer et al., versión que cuenta con unas características psicométricas adecuadas, al indicar un alfa de Cronbach para el puntaje global de 0,94 y superior a 0,72 en todas las subescalas (16). Este instrumento ha sido ampliamente utilizado en la población colombiana.

Los pacientes fueron captados en el servicio de consulta externa de forma posterior a la cita médica de control programada, y el instrumento fue autodiligenciado de forma supervisada por el auxiliar de investigación para resolver las dudas que pudieran presentarse durante el proceso. El cálculo del puntaje se realizó a través de la calculadora del cuestionario, la cual otorga un resultado que varía entre 0 y $100 \%$, donde un menor porcentaje es un indicativo de mayor calidad de vida.

Análisis de datos: los reportes de los pacientes, contenidos en los instrumentos aplicados, fueron trasladados a la calculadora del cuestionario y posteriormente verificados por los investigadores para evitar errores de transcripción, con ello se obtuvo la calificación total y por subescalas. El análisis descriptivo se realizó utilizando el programa SPSS versión 23, tanto en el cálculo de frecuencias como de porcentajes para el procesamiento de las características sociodemográficas y cálculos de mediana, media y desviación estándar para el análisis tanto de las subescalas como de la escala global.

Consideraciones éticas: se consideraron las normas nacionales e internacionales que rigen la investigación en salud, contenidas en la resolución 008430 de 1993, así como las directrices para la investigación en seres humanos propuestas por la Organización Mundial de la Salud y la Declaración de Helsinki. La investigación fue sometida y aprobada en el comité de ética e investigación de la institución en la cual se desarrolló el estudio. Los participantes firmaron el consentimiento informado, y se mantuvo la confidencialidad de su información. Se obtuvo la autorización de los autores del instrumento para su uso. 


\section{Resultados}

\section{Variables sociodemográficas}

La tabla 1 describe el valor de la estadística, las frecuencias y los porcentajes de las características sociodemográficas de los participantes. Se evidencia que la edad oscila entre 54 y 92 años, donde se observa que el $50 \%$ de los pacientes tienen 73 años o menos. Adicionalmente, predominan las mujeres, casadas, con formación primaria y secundaria, un nivel socioeconómico medio-bajo, pertenecientes a los estratos 2-3, y con una red de apoyo en donde prevalecen los hijos y la ausencia de educación en relación con la enfermedad.

\section{TABLA 1.}

Características de los participantes

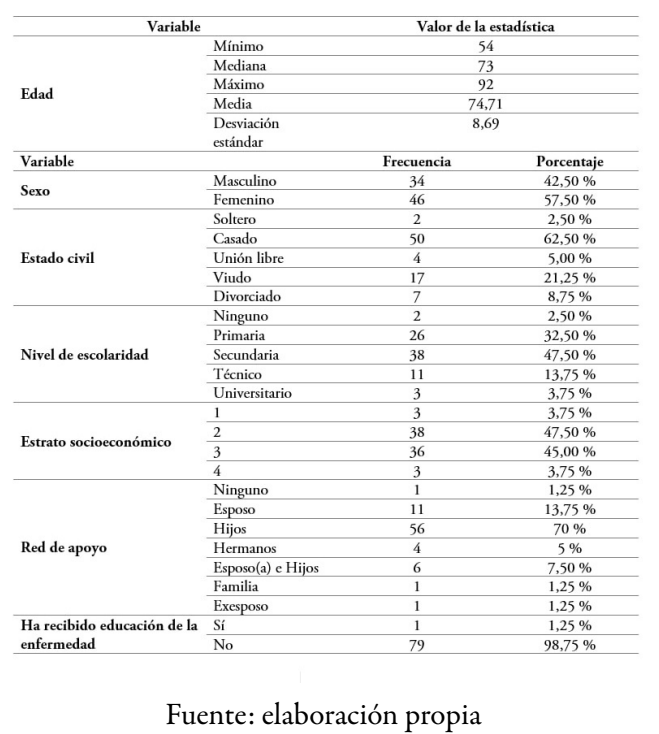

\section{Calidad de vida global y por subescalas}

La tabla 2 describe el comportamiento de la calidad de vida global, así como de las subescalas medidas con el SGRQ. Teniendo en cuenta que el resultado del instrumento es inversamente proporcional a la calidad de vida, se evidencia, en el puntaje global de la escala, una media hacia el límite inferior, lo que indica, en general, una adecuada calidad de vida en los participantes del estudio. Un comportamiento similar se observa en las subescalas de síntomas e impacto, sin embargo, la dimensión de actividad presenta una media cercana a 50, lo cual demuestra que esta es la más afectada por la enfermedad.

TABLA 2.

Calidad de vida global y por subescalas de los participantes

\begin{tabular}{lcccc}
\multicolumn{1}{c}{ Estadística } & Síntomas & Actividad & Impacto & Escala global \\
\hline Mínimo & 6,3245 & 12,3728 & 0 & 9,2392 \\
\hline Cuartil 1 & 24,9509 & 41,7253 & 10,9784 & 22,1305 \\
\hline Mediana & 35,8189 & 53,5274 & 19,1189 & 31,4433 \\
\hline Cuartil 3 & 54,5321 & 73,0112 & 40,5869 & 52,3437 \\
\hline Máximo & 82,3849 & 92,5068 & 69,6808 & 76,3159 \\
\hline Media & 38,7356 & 54,8546 & 25,6473 & 36,6737 \\
\hline Desviación estándar & 17,7528 & 18,6654 & 19,8100 & 17,8660 \\
\hline Asimetría & 0,2650 & 0,0843 & 0,8079 & 0,5561 \\
\hline
\end{tabular}

Fuente: elaboración propia

De forma adicional, es importante resaltar la versatilidad que se presenta en los reportes de los pacientes, tanto en las dimensiones como en la puntuación global. Esto permite evidenciar puntuaciones muy cercanas a 0 , así como puntajes por encima de 80 , lo que deja ver la variabilidad de las consecuencias que ejerce la 
enfermedad sobre la calidad de vida de los individuos con EPOC, tal como se evidencia en el gráfico de boxplot presentado a continuación.

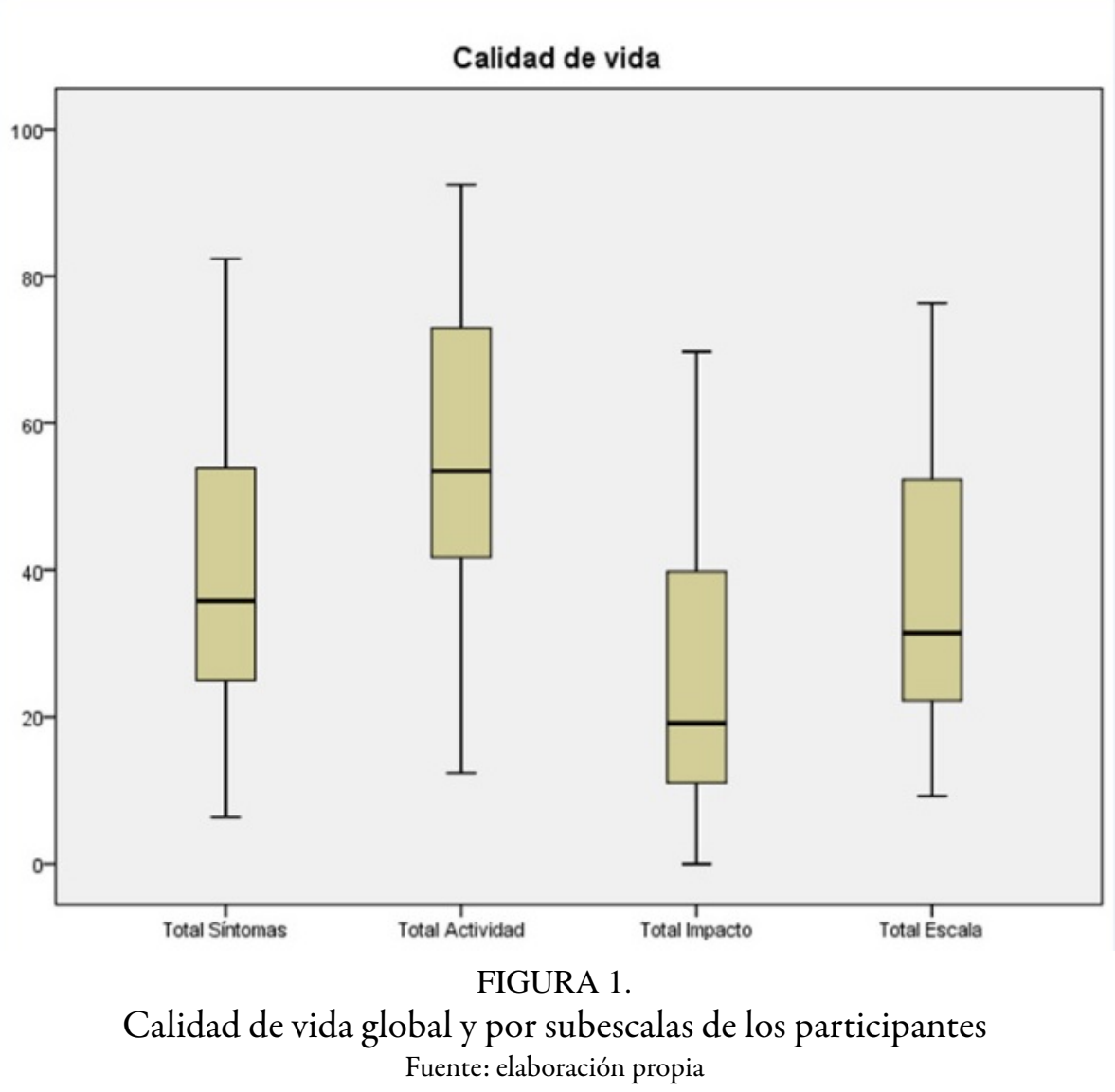

La mayoría de los pacientes describieron como "bueno" su estado de salud en el momento del diligenciamiento del instrumento. En relación con los síntomas, los participantes reportaron con mayor frecuencia la presencia de tos y sensación de falta de aire, en comparación con la presencia de secreciones y sibilancias. También manifestaron, en su mayoría, una crisis respiratoria en las últimas 4 semanas, con una duración inferior a un día, de forma que evalúan como "buenos" la mayoría de los días de la semana, y solo 9 de los 80 pacientes declararon empeoramiento de las sibilancias al levantarse en la mañana.

Con respecto a la actividad, la mayoría de los pacientes describieron sensación de falta de aire ante actividades como subir un piso por las escaleras, subir por una superficie empinada, y al agacharse, de manera que consideran que sus problemas respiratorios les obligan a caminar más despacio o detenerse y les impiden actividades en las cuales deben levantar objetos pesados.

En cuanto al impacto, la mayoría de los pacientes reportan que su estado respiratorio les genera pocos problemas y no han afectado los trabajos remunerados desarrollados, sin embargo, sienten pánico cuando no perciben un ingreso suficiente de aire y consideran que hacer ejercicio es arriesgado. Los pacientes manifiestan, en general, que su enfermedad respiratoria les impide hacer una o dos cosas que les gustaría.

\section{Discusión}

Algunos autores consideran que la EPOC se presenta con mayor frecuencia en los adultos mayores y resulta de un acumulo de factores de riesgo a lo largo del transcurso de la vida (11). El estudio de Betancourt-Peña et al. (17) reveló una edad media de 71 años, lo cual concuerda con los hallazgos de la presente investigación, en 
donde se evidenció una edad mínima de 54 años con una media de 74,7, datos coherentes con los reportados en la literatura nacional e internacional.

Por otra parte, algunos estudios demuestran que los hombres son más propensos a padecer la enfermedad, por lo que la prevalencia es mayor (18-20), sin embargo, esto no resulta similar a los resultados de este estudio, en donde las mujeres representan el 57,5 \% de la muestra. Cabe destacar que, si bien la literatura reporta el consumo de tabaco en el primer lugar de las causas asociadas con la EPOC, existen otras etiologías, como las de carácter genético y ambiental; en Colombia la exposición a biomasa, y con ello a contaminantes ambientales como lo es el humo de leña para la cocción de los alimentos, es frecuente en la población (21-23), lo que podría estar asociado con un incremento en las cifras que demuestran la prevalencia en el sexo femenino. De forma adicional, López-Campos (24) considera que el aumento del tabaquismo y el envejecimiento poblacional son situaciones especialmente relevantes en las mujeres, lo cual incrementa la prevalencia de la enfermedad en este grupo.

Por otra parte, diversos estudios revelan un predominio de pacientes con nivel educativo de primaria (25, 26), lo cual es coherente con los datos de esta investigación. Este aspecto podría guardar relación con el nivel socioeconómico de los participantes, ya que los ingresos insuficientes se asocian a un menor acceso a la educación, adicionalmente, existen otros factores que inciden en las bajas tasas de formación en Colombia, como una deficiente transición entre niveles, la deserción y el bajo acceso (27).

Con respecto a la calidad de vida global, Posada et al. (13) revelaron una media de $38 \pm 17$ en el SGRQ para los pacientes con EPOC en la ciudad de Bogotá, y demostraron así una calidad de vida similar a la de este estudio, sin embargo, a nivel internacional, los hallazgos difieren, pues Nonato et al. (28) hallaron una media de 52,3 + 19, lo que demuestra una peor calidad de vida.

Adicionalmente, la presencia de síntomas es una de las características más relevantes de la EPOC, tanto así, que la clasificación GOLD, actualizada en el 2019, contempla la evaluación de la disnea como como un criterio sintomático para la clasificación de la enfermedad (29). El presente estudio reveló un predominio de síntomas como tos y disnea, así como una media en la subescala de síntomas de 38,73 $\pm 17,75$, lo que indica una adecuada calidad de vida. No obstante, es necesario resaltar amplitud en el rango encontrado en la dimensión, en donde los puntajes oscilaron entre 6,32 y 82,3, datos que revelan la variabilidad en la presentación y magnitud de los síntomas que experimentan los pacientes. Estos resultados no se correlacionan con la investigación de Lutter et al. (30), en la cual la subescala de síntomas evidencia un reporte superior en la línea de base del estudio, donde arroja un puntaje de 54,4 $\pm 21,1$, lo que muestra una mayor repercusión de la disnea, la tos y las secreciones sobre el desempeño de estas personas.

Es bien conocido que la EPOC produce limitaciones en el desarrollo de la actividad física de los pacientes, aun cuando la enfermedad se encuentra en su estado más incipiente (31), por ello, la dimensión de actividad está frecuentemente afectada. El estudio realizado por Sánchez-Castillo et al. (32) reveló un nivel moderado de actividad física, asociado a la presencia de la patología. Pese a ello, las mujeres y las personas que se encuentran entre los 60 y 69 años desarrollan una menor cantidad de ejercicio semanal en comparación con el resto de la población estudiada, esto puede correlacionarse con los datos del presente estudio, en el cual se evidencia que la dimensión más comprometida es la de actividad, probablemente derivada de los cambios pulmonares y musculares que deterioran la funcionalidad de quien padece la enfermedad. Sin embargo, a pesar de ser la dimensión más afectada, los datos reportados en esta investigación $(54,85 \pm 18,66)$ son inferiores a los descritos en el estudio de Pineda-Higuita et al. (33), quienes encontraron en esta subescala, una media de 76,49 $\pm 21,22$.

Gran parte de los pacientes de este estudio consideran que hacer ejercicio es arriesgado, sin embargo, resulta relevante mencionar que la actividad física desempeña un papel protector para el paciente con EPOC, como un factor asociado a la reducción de la mortalidad, las exacerbaciones (34) y los reingresos hospitalarios (35), esto pone de manifiesto la importancia del tratamiento interdisciplinar, el entrenamiento y la educación por 
parte del profesional de enfermería en relación con la actividad física, como un aspecto determinante en la mejoría del estado de salud, y con ello en la calidad de vida $(36,37)$.

Cabe anotar que la EPOC provoca no solo limitaciones de tipo físico, sino también psicológico, las cuales, por su carácter progresivo, dificultan el normal desempeño de los individuos, y generan cierto grado de dependencia, que a su vez puede condicionar un deterioro en las relaciones sociales (25). No obstante, los resultados de la presente investigación revelaron una adecuada calidad de vida en la subescala de impacto, con una media de 25,64 $\pm 19,8$, que comprendía, incluso, un valor mínimo de 0 . Sin embargo, y de forma similar a lo ocurrido con las otras dimensiones, el puntaje máximo obtenido fue de 69,6, lo cual revela la magnitud que puede tener la enfermedad sobre el funcionamiento social y psicológico de los pacientes. Los resultados de esta investigación son coherentes con el estudio de Lutter et al. (30), en el cual la dimensión de impacto fue aquella que demostró la menor afección, que reveló un puntaje de 28,6 $\pm 19,8$, e indicó una mejor calidad de vida.

En relación con los hallazgos de la presente investigación, vale la pena resaltar el papel protagónico que desempeña el profesional de enfermería en la atención de pacientes con EPOC, en donde la valoración integral del sujeto y la determinación de aspectos como la calidad de vida, la magnitud de los síntomas, la tolerancia a la actividad física y el impacto de la enfermedad, se convierten en aspectos imprescindibles en la planeación del cuidado y, con ello, en el favorecimiento del control de la enfermedad.

\section{Conclusiones}

La calidad de vida relacionada con la salud es un indicador de las repercusiones que tiene la EPOC sobre la ejecución de las diferentes actividades físicas y sociales de los pacientes, por ello, su evaluación rutinaria y la identificación de las características sociodemográficas de los pacientes se convierten en un aspecto fundamental en la valoración de dicha población.

Los pacientes con EPOC demostraron poca afectación en la calidad de vida global, sin embargo, la subescala más comprometida fue la de actividad, y la menos afectada fue la de impacto, estos resultados revelan la imperante necesidad de innovar en el desarrollo de herramientas educativas que favorezcan el control de la enfermedad y minimicen el efecto sobre el desempeño de los pacientes en sus actividades cotidianas.

\section{Limitaciones}

Se presentan limitaciones en relación con el análisis que se hubiera podido desarrollar a partir de la clasificación de la gravedad de la patología, dado que no todos los pacientes contaban con reportes espirométricos recientes o reportaban incapacidad para completar la prueba.

\section{Referencias}

1. Global Initiative for Chronic Obstructive Lung Disease. Pocket guide to COPD diagnosis, management and prevention. A guide for health professionals [Internet]. 2020. Disponible en: https://goldcopd.org/wp-content /uploads/2020/03/GOLD-2020-POCKET-GUIDE-ver1.0_FINAL-WMV.pdf

2. Miravitlles M, Soler-Cataluña JJ, Calle M, Molina J, Almagro P, Quintano JA, et al. Spanish Guidelines for Management of Chronic Obstructive Pulmonary Disease (GesEPOC) 2017. Pharmacological Treatment of Stable Phase. Arch Bronconeumol [Internet]. 2017;53(6):324-335. https://doi.org/10.1016/j.arbres.2017.03. 018 
3. Martínez-Luna M, Rojas-Granados A, Lázaro-Pacheco R, Meza-Alvarado J, Ubaldo-Reyes L, Ángeles-Castellanos M. Enfermedad Pulmonar Obstructiva Crónica (EPOC). Bases para el médico general. Rev. Fac. Med. UNAM [Internet]. 2020;63(3):28-35. https://doi.org/10.22201/fm.24484865e.2019.63.3.06

4. Institute for Health Metrics and Evaluation. Global Burden of Disease Data visualizations [Internet]. 2017. Disponible en: https://vizhub.healthdata.org/gbd-compare/

5. Gil-Rojas Y, Torres-Duque CA, Figueredo MC, Hernández F, Castañeda-Cardona C, Lasalvia P, et al. Estimación de la prevalencia de EPOC en Colombia a partir del registro individual de prestaciones de servicios de salud (RIPS). Rev Colomb Neumol [Internet]. 2019;31(1). https://doi.org/10.30789/rcneumologia.v31.n1.2019.325

6. Johansson H, Berterö C, Berg K, Jonasson LL. To live a life with COPD. The consequences of symptom burden. Int J Chron Obstruct Pulmon Dis [Internet]. 2019;14:905-909. https://doi.org/10.2147/COPD.S192280

7. Dua R, Das A, Kumar A, Kumar S, Mishra M, Sharma K. Association of comorbid anxiety and depression with chronic obstructive pulmonary disease. Lung India [Internet]. 2018;35(1):31-36. https://doi.org/10.4103/lun gindia.lungindia_537_16

8. Miravitlles M, Ribera A. Understanding the impact of symptoms on the burden of COPD. Respir Res [Internet]. 2017;18(67):1-11. https://doi.org/10.1186/s12931-017-0548-3

9. Nguyen HT, Collins PF, Pavey TG, Nguyen NV, Pham TD, Gallegos DL. Nutritional status, dietary intake, and health-related quality of life in outpatients with COPD. Int J Chron Obstruct Pulmon Dis. 2019;14:215-226. https://doi.org/10.2147/COPD.S181322

10. Vázquez-Espinosa E, López-Riolobos C, Sampedro-Núñez M. Alteración nutricional del paciente con EPOC. Etiopatogenia. Consecuencias de la desnutrición en el paciente con EPOC. Rev Patol Respir. 2018;21(Supl. 2):S166-S171.

11. Augusti A, Celli B. Natural history of COPD: gaps and opportunities. ERJ Open Res. [Internet]. 2017;3(4). htt ps://doi.org/10.1183/23120541.00117-2017

12. Urzúa A. Calidad de vida relacionada con la salud: Elementos Conceptuales. Rev Med Chile. 2010;138(3):358-365. http://doi.org/10.4067/S0034-98872010000300017

13. Posada A, Caballero A, Ibañez M, Ardila L, Álvarez A, Soler S. Evaluación de la calidad de vida en pacientes del programa manejo integral de la enfermedad pulmonar obstructiva crónica (EPOC) de la EPS Sanitas en Bogotá. Rev. Méd Sanitas. 2009;12(2):14-21. Disponible en: https://revistas.unisanitas.edu.co/index.php/RMS/articl e/view/129/101

14. Betancourt-Peña J, Muñoz-Erazo B, Mora-Guerra R. Calidad de vida en pacientes con enfermedad pulmonar obstructiva crónica al ingreso de un programa de rehabilitación pulmonar. Rev. Col. Reh. 2015;14:96-102. Disponible en: https://revistas.ecr.edu.co/index.php/RCR/article/view/18/40

15. Bolívar-Grimaldos F, Cano-Rosales DJ, Duran-Sandoval JN, Albarracín-Ruiz MJ, Rincón-Romero K. Calidad de vida de pacientes con enfermedad pulmonar obstructiva crónica, participantes en un programa educativo integral. Rev Univ Ind Santander Salud [Internet]. 2019;51(4):301-307. https:// doi.org/10.18273/ revsal.v5 $\ln 4-2019003$

16. Ferrer M, Alonso J, Prieto L, Plaza V, Monsó E, Marrades R, et al. Validity and reliability of the St George's Respiratory Questionnaire after adaptation to a different language and culture: the Spanish example. Eur Respir J. [Internet]. 1996;9(6):1160-1166. https://doi.org/10.1183/09031936.96.09061160

17. Betancourt-Peña J, Artunduaga-Landazábal MC, Gil-Ramírez MP, León-Atoy K, Ávila-Valencia JC. Comparación de variables clínicas, capacidad funcional, ansiedad/depresión y calidad de vida en pacientes con EPOC de acuerdo a la clasificación GOLD 2018. Fisioterapia [Internet]. 2021;43(1):19-29. https://doi.org/10.1016/j.ft .2020 .06 .006

18. Martínez-Pérez JA, Vásquez-Marín CE, Rodríguez-Zapata M. Prevalencia de la enfermedad pulmonar obstructiva crónica en una zona rural de Guadalajara. Rev Esp Salud Pública. 2016;90:E13. Disponible en: https://scielo.i sciii.es/pdf/resp/v90/1135-5727-resp-90-e40002.pdf 
19. González-Rodríguez R, Barcón-Díaz L. Caracterización clínico-epidemiológica de pacientes con enfermedad pulmonar obstructiva crónica reagudizada. AMC. 2018;22(3):292-302. Disponible en: http://scielo.sld.cu/sci elo.php?script $=$ sci_arttext\&pid $=\$ 1025-02552018000300005$

20. Ayora A, Soler LM, Gasch, AC. Análisis de dos cuestionarios sobre la calidad de vida en pacientes con Enfermedad Pulmonar Obstructiva Crónica. Rev. Latino-Am. Enfermagem [Internet]. 2019;27:e3148. https://doi.org/10. $1590 / 1518-8345.2624 .3148$

21. Botero-Mesa S, Restrepo DA. Conceptos esenciales de la EPOC, prevalencia e impacto en América Latina. Med UPB. 2015;34(1):49-60. Disponible en: https://www.redalyc.org/pdf/1590/159046025007.pdf

22. Ezzati M. Indoor air pollution and health in developing countries. Lancet [Internet]. 2005;366(9480):104-106. h ttps://doi.org/10.1016/S0140-6736(05)66845-6

23. López-Campos JL, Fernández-Villar A, Calero-Acuña C, Represas-Represas C, López-Ramírez C, LeiroFernández V, et al. Exposición laboral y a biomasa en la enfermedad pulmonar obstructiva crónica: resultados de un análisis transversal del estudio On-Sint. Arch Bronconeumol. 2017;53(1):7-12. https://doi.org/10.1016 /j.arbres.2016.04.013

24. López-Campos JL, Tan W, Soriano JB. Global burden of COPD. Respirology [Internet]. 2016;21(1):14-23. htt ps://doi.org/10.1111/resp.12660

25. Fernández-García S, Represas-Represas C, Ruano-Raviña A, Mosteiro-Añón M, Mouronte-Roibas C, FernándezVillar A. Perfil social de los pacientes que ingresan por una agudización de EPOC. Un análisis desde una perspectiva de género. Arch Bronconeumol. [Internet]. 2020;56(2):84-89. https://doi.org/10.1016/j.arbres.20 19.03.009

26. Correa S, Gonzaléz M, De-Betolaza S, Spiess C, Perera P, Algorta S. Descriptive study of patients with COPD assisted in internal medicine of the Hospital Pasteur of Montevideo: demographic characteristics and comorbidities. Rev Urug Med Int. [Internet]. 2019;4(1):5-15. https://doi.org/10.26445/04.01.1

27. Organización para la Cooperación y el Desarrollo Económicos. Education in Colombia. Traducción por el Ministerio de Educación Nacional de Colombia [Internet]. 2016, pp. 29-30. Disponible en: https://www.min educacion.gov.co/1759/articles-356787_recurso_1.pdf

28. Nonato N, Díaz O, Nascimento O, Dreyse J, Jardim R, Lisboa C. Behavior of quality of life (SGRQ) in COPD patients according to BODE scores. Arch Bronconeumol. [Internet]. 2015;51(7):315-321. https://doi.org/10 $.1016 /$ j.arbr.2015.04.001

29. Global Initiative for Chronic Obstructive Lung Disease (GOLD). Global Strategy for the Diagnosis, Management, and Prevention of Chronic Obstructive Pulmonary Disease (Actualización 2019) [Internet]. 2019. Disponible en: www.goldcopd.org

30. Lutter J, Jörres RA, Kahnert K, Schwarzkopf L, Studnicka M, Karrasch S, et al. Health-related quality of life associates with change in FEV1 in COPD: results from the COSYCONET cohort. BMC Pulm Med. [Internet]. 2020;20(1):148. https://doi.org/10.1186/s12890-020-1147-5

31. Van Helvoort HA, Willems LM, Dekhuijzen PR, Van Hees HW, Heijdra YF. Respiratory constraints during activities in daily life and the impact on health status in patients with early-stage COPD: a crosssectional study. NPJ Prim Care Respir Medicine. 2016;26:16054. https://doi.org/10.1038/npjpcrm.2016.54

32. Sánchez-Castillo S, Cepeda-Quintanar S, Díaz-Suárez A, Smith L, López-Sánchez GF. Actividad física en personas con EPOC residentes en España: Diferencias según sexo y edad. J Sports Med. 2019;11(Supl. 1):59-68. Disponible en: https://dialnet.unirioja.es/servlet/articulo?codigo=7023959

33. Pineda-Higuita SE, Ramos-Melchor V, Cadavid-Carmona D. Calidad de vida en pacientes con Enfermedad Pulmonar obstructiva crónica. 2014. Rev Univ. Salud [Internet]. 2016;18(3):482-493. http://doi.org/10.2226 $7 /$ rus. 161803.53

34. Dueñas-Espín I, Demeyer H, Gimeno-Santos E, Polkey M, Hopkinson N, Rabinovich R, et al. Depression symptoms reduce physical activity in COPD patients: a prospective multicenter study. Int J Chron Obstruct Pulmon Dis. 2016;11:1287-1295. https://doi.org/10.2147/COPD.S101459 
35. Demeyer H, Burtin C, Hornikx M, Camillo CA, Van Remoortel H, Langer D, et al. The minimal important difference in physical activity in patients with COPD. PLoS ONE [Internet]. 2016;11(4):e0154587. https://d oi.org/10.1371/journal.pone.0154587

36. Esteban C, Quintana JM, Aburto M, Moraza J, Egurrola M, Pérez-Izquierdo J, et al. Impact of changes in physical activity on health-related quality of life among patients with COPD. Eur Respir J. 2010;36(2):292-300. https: //doi.org/10.1183/09031936.00021409

37. Gotera-Rivera C, Simón-Rodríguez B, Salgado-Aranda S. Actualización de las guías clínicas en la EPOC. Rev Patol Respir. 2019;22(Supl. 2):S179-S186. Disponible en: https://www.revistadepatologiarespiratoria.org/descargas /PR_22-S2_S179-S186.pdf

\section{Notas}

* Artículo original de investigación. Financiamiento: Pontificia Universidad Javeriana

\section{Licencia Creative Commons CC BY 4.0}

Cómo citar este artículo: Achury-Beltrán LF, García-Peñuela P. Calidad de vida del paciente con enfermedad pulmonar obstructiva crónica. Investig Enferm Imagen Desarr. 2021;23. https://doi.org/10.11144/Javeria na.ie23.cvpe 